\title{
16a-Bromoepiandrosterone as a new candidate for diabetes-tuberculosis comorbidity treatment
}

\author{
Manuel Othoniel Lopez Torres ${ }^{1}$, Brenda Marquina ${ }^{2}$, Octavio Ramos-Espinosa ${ }^{3}$, Dulce \\ Mata $^{1}$, Jorge Barrios-Payan ${ }^{4}$, Guillermina Baay ${ }^{5}$, Sara Huerta-Yépez ${ }^{6}$, Cristian Alfredo \\ Segura-Cerda ${ }^{7}$, Estela Bini ${ }^{8}$, Ivan Torre-Villalvazo ${ }^{1}$, Nimbe Torres ${ }^{1}$, Armando R Tovar ${ }^{1}$, \\ William Chamberlin ${ }^{9}$, Yu Ge ${ }^{9}$, Andrea Carranza ${ }^{10}$, and Rogelio Hernandez Pando ${ }^{2}$ \\ ${ }^{1}$ Instituto Nacional de Ciencias Medicas y Nutricion Salvador Zubiran \\ ${ }^{2}$ National Institute of Medical Sciences and Nutrition \\ ${ }^{3}$ National Institute of Medical Sciences and Nutrition Salvador Zubirán \\ ${ }^{4}$ National Institute of Medical Sciences and Nutrition "Salvador Zubiran" \\ ${ }^{5}$ Oncological Diseases Research Unit, Hospital Infantil de Mexico "Federico Gomez", \\ Mexico City, Mexico. \\ ${ }^{6}$ Hospital Infantil de Mexico "Federico Gomez" \\ ${ }^{7}$ Centro de Investigacion y Asistencia en Tecnologia y Diseno del Estado de Jalisco \\ ${ }^{8}$ National Institute of Medical Sciences and Nutrition "Salvador Zubirán" \\ ${ }^{9}$ Protibea Therapeutics \\ ${ }^{10}$ Universidad de Buenos Aires
}

October 27, 2020

\begin{abstract}
Being the leading cause of mortality by a single infectious agent, tuberculosis (TB) continues as one of the most relevant issues of public health. Another pandemic disease is type 2 diabetes mellitus (T2D) is usually associated with immunodeficiency. Thus, an increased prevalence of TB-T2D comorbidity represents one of the most significant challenges for health providers. During the chronic phase of both diseases, several immunoendocrine abnormalities are occurring, but extra-adrenal production of active glucocorticoids (GCs) is a possible deleterious factor in both entities. Active GCs have been related to insulin resistance and suppression of Th1 responses, contributing to T2D and TB pathogenesis. 11- $\beta$-hydroxysteroid dehydrogenase type 1 (11$\beta H S D 1)$ catalyzes the conversion of inactive GGs in their active form (cortisol or corticosterone in rodents) in the lungs and liver and could be responsible for this immunoendocrine disfunction. Dehydroepiandrosterone (DHEA) is an anabolic adrenal hormone with antagonist effects against GCs on immune cells and glucose metabolism. A synthetic analog of DHEA, the 16abromoepindrosterone (BEA), lacks an anabolic effect while keeping his immune and metabolic effect. The therapeutic efficiency of BEA was studied in a murine model of T2D-TB comorbidity. TB-T2D mice underwent more severe lung disease than in TB-infected but non-diabetic animals. BEA decreased the active form of GCs and 11- $\beta$ HSD1 expression, while increasing 11-ßHSD2 expression, which reduced hyperglycemia and liver steatosis, lung bacillary loads, and pneumonia. Thus, it seems that BEA is an efficient therapy to control metabolic and immune abnormalities caused by high active GCs production.
\end{abstract}

\section{Introduction}

Type 2 Diabetes (T2D) is currently the most widespread susceptibility factor for tuberculosis (TB) with a high incidence and prevalence in low- and middle-income countries (1). In most cases, Mycobacterium tuberculosis (Mtb) infection is controlled by immune mechanisms that contribute to granuloma development 
and control of bacterial growth (2). However, recent research on TB-T2D comorbidity shows that innate and adaptive immunity are more affected when both conditions occur together than when only one disorder is present. (3).

A type $1 \mathrm{~T}$ helper (Th1) cell response mediates the protective immune response to TB through macrophages activation by IFN $-\gamma$ and TNF $\alpha$ (4). Observations in T2D patients show an impaired response to Mtb infection (5), consisting in alterations in bacterial recognition, phagocytosis, and a deficient immune cellular response with low cytokine and chemokine production (6), resulting in severe TB manifestations (7).

A new aspect in understanding the higher susceptibility of T2D to develop TB is the study of the abnormal metabolic factors and neuro-immune-endocrine regulation in this co-morbidity. In homeostatic conditions and under acute or chronic stress, the principal source of glucocorticoids (GCs) are the adrenal glands regulated by the activation of the Hypothalamus-Pituitary-Adrenal (HPA) axis (8). Besides this source of circulating GCs, there is a local organ conversion of inactive to active GCs (9), which is mediated by the enzyme 11- $\beta$-hydroxysteroid dehydrogenase type 1 (11- $\beta$ HSD1) that has an oxo-reductase activity using $\operatorname{NADP}(\mathrm{H})$ as a cofactor. This enzyme converts inactive cortisone to active cortisol in humans or corticosterone in rodents (10). Interestingly, there is another enzyme with the opposite effect. 11- $\beta$-hydroxysteroid dehydrogenase type 2 (11- $\beta$ HSD2) acts exclusively as an NAD-dependent dehydrogenase that converts active cortisol or active corticosterone to inactive cortisone (11).

Although blood levels of glucocorticoids are in the normal range in T2D and TB, it seems that this is not

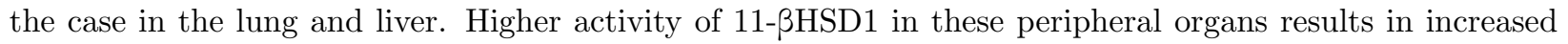
$\mathrm{GC}$ production in these organs.

GCs are critical homeostatic hormones that are overproduced after diverse environmental insults, and in general they have a negative effect on metabolism and the immune response (12). GCs exert their effects by binding to glucocorticoid receptors (GR) present in all cell types. Hence, GR signaling plays an essential role in the modulation of many biological functions in immune cells and several tissues, including liver, lung, adipose tissue, thymus and muscle (13).

Cortisol suppresses the expression of pro-inflammatory and adhesion molecules, thus preventing the extravasation of neutrophils to the site of inflammation (13). Chronic exposure induces an anti-inflammatory gene expression profile of resident macrophages and decreases their phagocytic activity. Furthermore, active GCs suppress Th1 cytokine production and induce cell death (14), promoting a Th2 environment that impairs granuloma formation (7). GCs inhibit the proliferation of effector T cells and induce apoptosis of neutrophils, basophils, and eosinophils resulting in reduced inflammation (15). GCs also have an important contribution in the physiopathology of TB. It has been reported in an experimental model of progressive TB that at the time of maximal protective activity mediated by IFN- $\gamma, \mathrm{TNF}-\alpha$ and NO production, the hypothalamicpituitary-adrenal axis is activated, thereby stimulating the adrenal glands to secrete GCs (corticosterone) with the apparent aim of avoiding tissue damage produced by excessive lung inflammation. However, the excess of corticosterone also inhibits Th1 lymphocytes activity and induces differentiation of Th-2 lymphocytes. This favors bacterial survival and proliferation, causing animals death (14). As far as we know, there

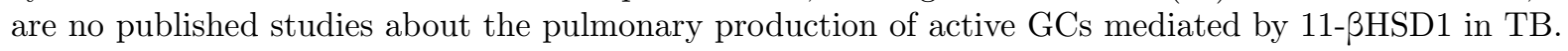

In metabolic diseases such as obesity and metabolic syndrome, GCs play a role in the development of diabetes, but interestingly, GCs in the circulation have the same concentrations as healthy subjects (16). Experimental

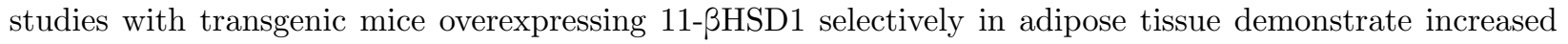
local levels of corticosterone that induce visceral obesity development, insulin resistance, diabetes, and hyperlipidemia (16). The increased conversion of active glucocorticoids in visceral adipose tissue increases lipolysis and fatty acid levels (17).

Moreover, active GCs are potent inducers of glycogenolysis and gluconeogenesis, resulting in a hyperglycemic state, deteriorating the condition of diabetic patients (18). 11- $\beta$ HSD1 also increases glucose output by activating phosphoenolpyruvate kinase (PEPCK) gene expression in the liver (19). Thus, active GCs have direct implications in the abnormal metabolic control and chronic complications of T2D (20). 
Regulation of cortisol is mediated by dehydroepiandrosterone (DHEA), an anabolic hormone of the adrenal cortex involved in the conversion of sexual steroids. This hormone displays antagonistic activity to cortisol, impacting the immune response (21) and glucose metabolism. The cortisol-DHEA ratio is modified in T2DTB comorbidity and represents a deleterious factor for both diseases (8). 16 $\alpha$-Bromoepiandrosterone (BEA) is a synthetic analog hormone of DHEA that modulates immune and metabolic responses $(22,23)$. Compared to DHEA, BEA does not display anabolic activities.

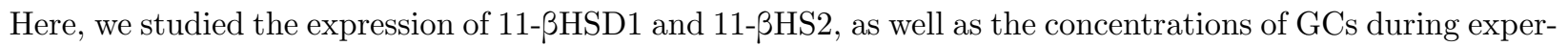
imental late TB and T2D/TB in the lung and liver and evaluate the therapeutic effect of BEA administration in a murine model of T2D-TB comorbidity.

\section{Materials and Methods}

\section{Ethics Statements}

All the animal work was done according to the guidelines of the Mexican law NOM 061-Z00-1999 and approved by the Internal Committee for the Care and Use of Laboratory Animals (CICUAL) of the National Institute of Medical Sciences and Nutrition in México.

\section{Experimental Model of Type 2 Diabetes and Progressive Pulmonary TB in BALB/c Mice}

To induce T2D, a non-genetic mouse model of non-insulin-dependent diabetes mellitus previously reported was reproduced (26). Briefly, young male BALB/c mice three weeks old were fed with a high fat diet (HFD) at $45 \%$ of calories during the whole course of the experiment. At 3 months old, animals received a single low dose $(100 \mathrm{mg} / \mathrm{kg}$ ) of estreptozotocin (STZ) ChemCruz (Santa Cruz Biotechnology, Dallas, TX) by intraperitoneal (i.p) injection. That STZ dose did not cause diabetes in chow-fed mice (26). We assessed the combined effect of HFD and STZ in glucose blood levels with Accu-chek active (Roche diabetes care Mannheim, Germany) test strips, after and before of STZ administration. Serum cholesterol and triglyceride concentrations were measured with Acu-trend one month after STZ administration.

After one month of STZ administration, animals showed hyperinsulinemia and hyperglycemia that reverted by the treatment with oral hypoglycemic drug (metformin), a similar metabolic pattern and respond to treatment of T2D (Fig. 1). Animals with these metabolic abnormalities compatible with T2D were infected by the intra-tracheal route with a high dose of Mtb reference strain H37Rv (ATCC No. 25618), in order to induce progressive pulmonary TB. Briefly, Mtb strain H37Rv was grown in Middlebrook 7H9 broth (DIFCO) supplemented with $0.2 \%$ glycerol, $10 \%$ OADC enrichment, and $0.02 \%$ Tween- 80 and maintained at $37^{*} \mathrm{C}$ in agitation. Mid log-phase cultures were used for infection. Mycobacteria were counted and stored at $-80^{*} \mathrm{C}$ until use. Bacterial aliquots were thawed and pulse-sonicated to remove clumps. For the infection, groups of T2D and control animals fed with chow diet were anesthetized in gas chamber using Sevofluorane and infected intra-tracheally with $2.5 \mathrm{~A}^{\sim} 10^{5}$ live bacilli using a cannula inside a biosafety level III cabinet. Mice were maintained in vertical position until spontaneous recovery and maintained in groups of five in cages fitted with micro-isolators connected to negative pressure in animal biosafety level III facilities.

In order to compare the evolution of TB in T2D and non-diabetic mice, groups of 5 mice were euthanized by exsanguination under pentobarbital anesthesia after 14,21, 28 and 120 days after infection. Lungs were rapidly collected and immediately frozen by immersion in liquid nitrogen and used for bacillary loads determination by colony forming units quantification and TNF $\alpha$ expression determined by RT-PCR, as described below. Ten animals per group were left untouched and their survival was recorded to construct survival curves.

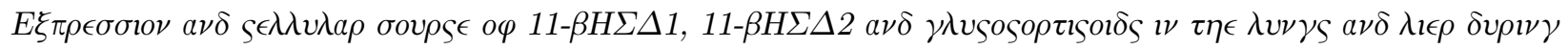
$\lambda a \tau \epsilon$ a $\tau \iota \epsilon \pi \nu \lambda \mu о \nu а \rho \psi T B$

Groups of five mice with T2D, TB and T2D/TB were euthanized by exsanguination under pentobarbital anesthesia after 60 and 120 days of infection, in two independent experiments. The late active disease is well established by this time in this model, as characterized by extensive pneumonia, high bacillary loads 
and low expression of IFN- $\gamma$ and TNF- $\alpha$. Three right lungs were intra-tracheally perfused with absolute ethanol and after $24 \mathrm{hr}$ the tissue was embedded for histology and immunohistochemistry studies, while the left lungs were immediately frozen for total RNA isolation and gene expression determination of 11- $\beta$ HSD1 and 11-3HSD2 by RT-PCR. The same tissue samples for the histology study were used to determine by immunohistochemistry the cellular source of corticosterone and cortisone and their concentrations by digital pathology. Tissue fragments from the liver were processed in the same way.

\section{BEA treatment in infected animals with and without T2D}

After two months of infection, treatment with BEA was started in groups of mice with TB or T2D/TB administrated each other day $.02 \mathrm{mg} / \mathrm{kg}$ by intra-tracheal route to produce a direct effect on the infected lung, and the same amount of BEA administrated by subcutaneous route for systemic distribution promoting hepatic effect. TB group received BEA only by intratracheal instillation. After 30 and 60 days of treatment, groups of six animals were euthanized by exsanguination under anesthesia with $210 \mathrm{mg} / \mathrm{kg}$ of intraperitoneal pentobarbital, inside in a cabinet of biosecurity level III. Three left lungs per time were perfused with absolute ethanol, fixed, and prepared for histopathological studies. After eliminating hilar lymph nodes and thymic tissues, five lungs were frozen and kept to $-80 * \mathrm{C}$ for bacilli load quantification and three lungs were used to determine the expression of diverse genes by RT-PCR. Two independent experiments were performed. Animals were monitored daily and humanely euthanized if exhibited respiratory insufficiency or substantial weight loss.

\section{Determination of colony-forming units in lung}

Right lungs from four mice at each time-point, in two different experiments, were used for bacillary load determination by colony forming counting (CFU) quantification. Lungs were homogenized with (MP Biomedicals FastPrep-24) in $1 \mathrm{~mL}$ of $0.05 \%$ Tween- 80 and the same methodology was use with spleens after weighted them. Four consecutive logarithmic dilutions were made from this homogenate. Ten microliters of each dilution were plated in duplicate on Bacto Middlebrook 7H10 agar (Difco, Detroit, MI, USA) enriched with OADC. Plates were incubated at $37^{\circ} \mathrm{C}$ and $5 \% \mathrm{CO} 2$ during 21 days for $\mathrm{CFU}$ quantification.

Preparation of lung tissue for histological and immunohistochemistry analysis

Lungs of mice were perfused with absolute ethanol by endotracheal route and fixed for $24 \mathrm{~h}$ and then embedded in paraffin blocks. Sections of $4 \mu \mathrm{m}$ width were obtained, mounted on glass slides, deparaffinized and stained with hematoxylin and eosin. For quantification and morphometric analysis determining the surface area affected by pneumonia, three different mouse lungs per time point were evaluated in two independent experiments.

For immunohistochemistry, lung tissue was sectioned and mounted on charged glass slides and then deparaffinized. Slides were first blocked for unspecific activity of peroxidase with methanol peroxide $3 \%$ for $1 \mathrm{~h}$. For

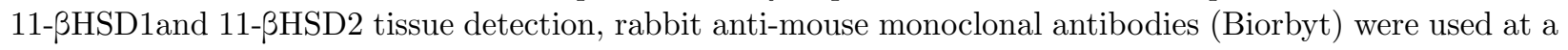
concentration of 1:250, incubated overnight in agitation, followed by incubation with secondary anti-rabbit IgG labeled with peroxidase. For cortisone and corticosterone immunostaining, rabbit antimouse monoclonal antibodies (LsBio) were used at a final concentration of 1:250. In both cases, bound antibodies were detected with diaminobenzidine and counterstained with hematoxylin.

Immunohistochemistry stained sections were analyzed by digital pathology. Briefly, stained sections were digitized at $200 \mathrm{x}$ magnification using an Aperio ScanScope CS (Aperio). The Aperio ScanScope CS images had a spatial resolution of $0.45 \mu \mathrm{m} /$ pixels. The images were analyzed using an ImageScope (Aperio). All the lung tissue sections were circumscribed and sent for automated image analysis using the Spectrum Software V11.1.2.752 (Aperio). For immunostaining GCs intensity quantification, an algorithm was developed to determine the total lung expression of cortisone and corticosterone in the whole tissue section (26-28).

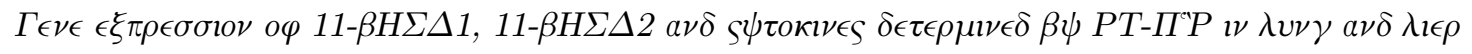

The right lungs of three mice per time point were rapidly obtained and stored in 1.5-ml cryotubes, imme- 
diately frozen in liquid nitrogen and maintained at $-80^{*} \mathrm{C}$ until processing. For homogenization, lungs were slowly defrosted, and zirconium flint beads were added to each tube and lung tissue was homogenized in the FastPrepR-24 (MP Biomedicals). RNA extraction was performed with the RNeasyR Mini kit (Qiagen) following the manufacturer's instructions.

Total RNA was quantified by spectrophotometry (A260/280), $100 \mathrm{ng}$ of RNA from each lung was used for the production of cDNA by retro-transcription, following the indications of the Omniscript kit (Qiagen), and later an endpoint PCR was run to amplify the constitutive housekeeping gene RPLP0 (Ribosomal protein large P0, Gen ID:11837, GenBank, NCBI) and its integrity was analyzed by running at $2 \%$ agarose gel stained with SYBR green.

Complementary DNA (cDNA) obtained from each sample was analyzed by real-time PCR (qPCR) using the Real-Time PCR system 7500 (Applied Biosystems) and the Quantitech SYBR Green Mastermix kit

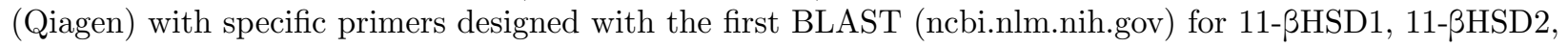
IFN- $\gamma$ and TNF- $\alpha$.

Regarding to relative quantification of $11-\beta H S D 1,11-\beta H S D 2$, the Ct values were determined by 7500 realtime PCR system (Applied Biosystems, Foster City, CA, USA), and the fold change of gene expression was calculated by the 2-(t) method (29). For absolute quantification, the number of copies of each target gene was normalized to 1 million amplicons of mRNA of the housekeeping gene RPLP0, including the standard

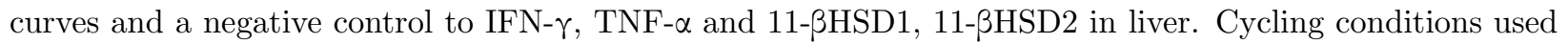
were as follows: initial denaturation at $95^{*} \mathrm{C}$ for $15 \mathrm{~min}$, followed by 40 cycles at $95^{*} \mathrm{C}$ for $20 \mathrm{~s}, 60^{*} \mathrm{C}$ for 20 $\mathrm{s}$, and $72 * \mathrm{C}$ for $34 \mathrm{~s}$.

\section{Statistical Analysis}

All the statistical analysis was performed using GraphPad Prism Software (version 6.0, La Jolla, USA). The data were analyzed using paired t-test, one- and two-tailed ANOVA with Bonferroni correction for multiple comparisons. P-values $<0.05$ were considered significant.

\section{Results}

\section{Comparative pulmonary disease evolution of TB \& T2D/TB}

Mice fed with hypercaloric diet for three weeks old and treated at eight weeks old with only one low dose of streptozotocin showed progressive weight increase (Fig. 1B), and after two months showed significant higher sera concentration of glucose, triglycerides, cholesterol and insulin (Fig. 1C, D, E, and F). The histology study of the liver showed extensive lipid cytoplasmic vacuolization of hepatocytes (steatosis) (Fig. 1H). Treatment of these mice with metformin or glibenclamide resulted in normal blood concentrations of glucose (Fig. 1G). Thus, these mice showed the metabolic parameters of T2D.

In comparison with chow fed mice infected with Mtb, infected T2D mice showed significant higher pulmonary bacilli burdens (Fig. 2A). T2D/TB mice also showed lower expression of TNF- $\alpha$ and decreasing survival rate than TB mice (Fig. 2B, and 2C). Thus, T2D/TB mice suffered more severe disease than TB mice.

\section{Comparative expression of GC's and GC's converting enzymes in the late phase of TB \& T2D-TB lungs}

Before evaluating the therapeutic effect of BEA, comparison among non-infected control mice fed with chow diet, T2D, TB and T2D-TB groups was done in the lungs of animals with late disease at day 120 of infection as determined by immunohistochemistry, the presence of corticosterone (active GC) and cortisone (inactive GC), as well as the genetic expression of the GC converting enzymes determined by RT-PCR and their cellular source by immunohistochemistry (Fig. 3). The lungs of control non-infected mice showed occasional bronchial epithelial cells with corticosterone positive immunostaining, while in T2D mice a clear increase in the number and intensity of corticosterone was seen in airways epithelium (Fig. $3 \mathrm{~A}$ ). In the TB and T2DTB groups the bronchial epithelium was negative, and the pneumonic areas showed intense corticosterone immunostaining in some lymphocytes and numerous macrophages, being many of them vacuolated or foamy 
macrophages (Fig. 3A). In comparison with the control non-infected group, there was also a significant

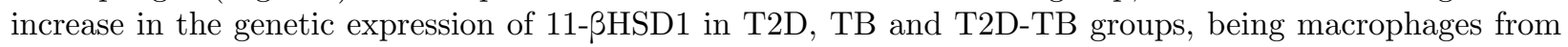
pneumonic areas the cells that showed strong immunostaining to this enzyme (Fig. 3B). Thus, there is a high expression of 11- $\beta$ HSD1 and corticosterone production in the inflammatory cells of pulmonary TB, which is even higher in mice with T2D-TB.

Regarding inactive GC cortisone, the lungs from non-infected mice showed positive immunostaining in the alveolar epithelium, which was lower in T2D animals and quite strong in the TB lungs, while in T2D-TB was slightly positive, almost negative (Fig. 3C). These immunohistochemical results are well correlated with the 11-ßHSD2 gene expression, which showed significant higher expression in non-infected lungs, while it was lower in TB, and the T2D-TB group showed the lowest (Fig. 3D).

Effect of BEA in lung and spleen bacillary burdens, pulmonary cytokine expression and tissue damage in TB and T2D-TB mice

To evaluate the therapeutic effect of BEA, the TB and T2D-TB groups and their respective control groups that received only the vehicle were compared through bacillary loads in the lung and spleen (CFUs), extent of tissue damage (percentage of lung surface affected by pneumonia), and the gene expression of the protective cytokines TNF- $\alpha$ and IFN- $\gamma$ (RT-PCR). In comparison with their respective controls non-treated mice, both T2D-TB and TB groups after one and two months of treatment with BEA showed significant reduction of pulmonary bacillary burdens (Fig. 4A). BEA induced an increase in gene expression of IFN- $\gamma$ and TNF- $\alpha$ after one month of treatment in both groups, and IFN- $\gamma$ in TB group after two months of treatment (Fig. $4 \mathrm{~B}$, and $4 \mathrm{C}$ ); as well as reduction of pneumonia. Interestingly, BEA treatment for two months induced in the spleen significant reduction of CFUs and weight increase only in the T2D-TB group (Fig. 4E, and 4F), which suggest spleen hyperplasia with better control of bacillary dissemination in this experimental group.

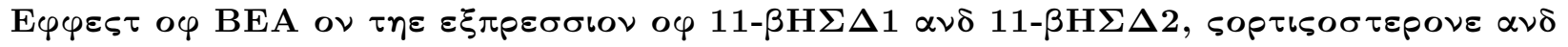

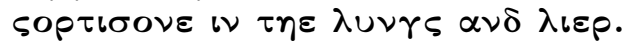

In the lung, treatment with BEA in TB and T2D-TB groups induced significant lower expression of 11BHSD1 after one and two months of treatment when compared with the control group (Fig. 5). This effect was even more evident in the liver of T2D-TB group, which also showed complete disappearance of steatosis and hyperglycemia (Fig. 6D).

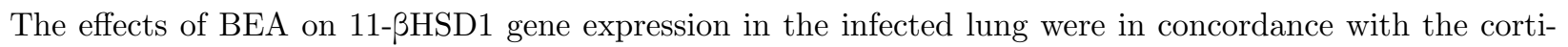
costerone immunehistochemistry staining, and its quantitative evaluation by digital pathology (Fig. 5A). BEA significantly decreased active corticosterone in TB and TB/T2D mice, being more pronounced in the TB group (Fig. 5C). In contrast, the expression of 11- $\beta$ HSD2 after one and two months of BEA treatment showed a significant increase in the TB group, and cortisone was increased in both groups (Fig. 5D, and $5 \mathrm{E})$.

Thus, it seems that BEA is a regulator of the genetic expression of these enzymes in the lung and liver,

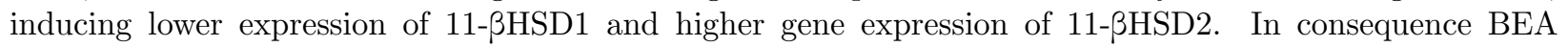
induced lower production of active corticosterone and higher production of inactive cortisone, which could contribute to increase the expression of the pro-inflammatory cytokines TNF- $\alpha$ and IFN- $\gamma$ decreasing the pulmonary bacillary loads. In the liver BEA improved lipid and glucose metabolism and normalized liver histology and glucose blood concentrations.

Thus, BEA improved the immune response and metabolism of the comorbidity $\mathrm{T} 2 \mathrm{D} / \mathrm{TB}$, suggesting that it could be a potential new immune therapeutic agent with beneficial immune, endocrine and metabolic activities.

\section{Discussion}

TB-T2D comorbidity is a new area of interest due to its recent increased incidence worldwide. T2D is a metabolic endocrine disease associated with abnormal secretion and action of insulin and chronic hyper- 
glycemia affecting multiple organ systems. T2D compromises host immune responses and impairs host ability to control microbial infections. Indeed, T2D produced several abnormalities in diverse immune mechanisms against Mtb that worsens the course of the disease requiring longer courses of chemotherapy $(7,8)$. Our results confirm the more aggressive course of TB in an experimental model of T2D/TB; and for the first time

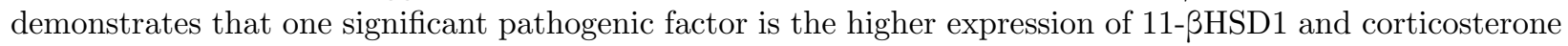
production during late advanced disease in the lungs and liver. The treatment with BEA efficiently decreases

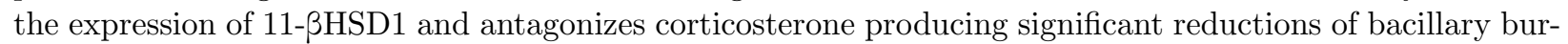
dens and hyperglycemia. These results suggest that this synthetic hormone can contribute to the treatment of this co-morbidity.

Mtb affects mainly the lungs producing chronic and excessive inflammation in which innate and adaptive immunity are deeply affected $(2,3)$. Infection starts by inhalation of saliva droplets with Mtb that are engulfed by alveolar macrophages, which are key cells in bacilli elimination and together with dendritic cells process $\mathrm{Mtb}$ antigens that are presented to $\mathrm{T}$ lymphocytes in regional lymph nodes. Lymphocytes migrate to the lung and with macrophages and other cells form containment structures known as granulomas which are the histopathological hallmark of TB (5). In early stages of active infection, Th1 cellular immune responses are protective, as IFN- $\gamma$, TNF $\alpha$ and other cytokines such as IL-12 induce macrophage activation, allowing bacterial growth control. Nevertheless, during late active disease, extensive inflammation produces a shift toward anti-inflammatory responses, such as Th- 2 cytokine pattern in which IL-4, IL-13 and other anti-inflammatory cytokines such as IL-10 and transforming growth factor- $\beta$ (TGF- $\beta$ ) induce a local immunosuppressive/anti-inflammatory milieu, resulting in poor containment of infection and progression of tissue damage. Mortality rates approximate 1\%. (30). Interestingly, besides these immunologic responses, there is an intense neuroendocrine response that creates a complex network of cytokines, hormones, and neurotransmitters that influence the outcome of TB pathogenesis $(8,9,31)$. These neuroendocrine responses include abnormal production of several hormones, that is partly mediated by the cytokines released during the immune response to Mtb (pro-inflammatory mediators), and also contribute to modify the central nervous system (CNS) response by activating the two major stress systems, the HPA axis (32) and the sympathetic nervous system (SNS) $(7,33)$.

HPA and GCs have an important contribution to the physiopathology of TB $(34,35)$. It was reported in the experimental model of progressive $\mathrm{TB}$ in $\mathrm{BALB} / \mathrm{c}$ mice that at the time of maximal protective activity mediated by IFN- $\gamma$, TNF- $\alpha$, IL-1 $\beta$ and NO production (day 21 after infection), pro-inflammatory cytokines such as TNF- $\alpha$ and IL-1 $\beta$ strongly activate the HPA axis, producing high expression of CRF in the hypothalamus and adrenal hyperplasia with high serum concentrations of corticosterone (32). Then, during the chronic or late phase after 28 days of infection, there is progressive adrenal atrophy and a corticosterone decrease in sera co-existence with extensive pneumonia (32). Thus, there is high GCs adrenal production apparently with the aim to avoid tissue damage produced by excessive lung inflammation. During the late phase of the disease when adrenal glands are atrophic, it seems that there is another source of corticosterone available by production in peripheral macrophages.

Our results show, for the first time, that this extra-adrenal source of GCs are macrophages, particularly foamy cells which strongly express the enzyme 11- $\beta$ HSD type 1 and serve as a new source of corticosterone in the inflamed tuberculous lung. This local source of corticosteroids acts in an autocrine and paracrine manner. However, this local excess of corticosterone also inhibits Th1 lymphocyte activity and induces differentiation of Th-2 lymphocytes that favors bacterial survival and proliferation causing animals death (14). Thus, this local anti-inflammatory activity mediated by high production of corticosterone in affected organs during active late TB have deleterious effect. Antagonistic hormones such as DHEA can restore balance to the system.

DHEA is the most abundant product of the human adrenal gland after puberty with maximum levels occurring in the late 20s. Its production then falls steadily with increasing age. DHEA has anti-glucocorticoid properties in several systems. For example, DHEA shows opposite effects to GCs on enzyme expression in the liver in obesity (1) and in the immune system (4). In a previous study, treatment with DHEA starting 
on day 60 after Mtb infection in BALB/c mice reduced the pulmonary bacillary burden and decreased tissue damage by reactivating the Th-1 cytokine response (36). In humans a deficit in DHEA relative to cortisol can be striking in severe human TB (8). However, DHEA is suboptimal for human use, partly because it is metabolized into sex steroids.

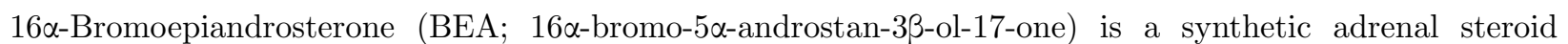
derivative that does not enter sex steroid pathways and when tuberculous BALB/c mice were treated with BEA, every other day beginning on day 60 , it produced a significant inhibition of bacterial proliferation and higher expression of protective cytokines (TNF- $\alpha$, IFN- $\gamma$ ). Moreover, when given as an adjunct to conventional chemotherapy, BEA enhanced bacterial clearance (36). Interestingly, this synthetic hormone showed therapeutic benefit in patients with TB (2), malaria (37) and in the Acquired Immune Deficiency Syndrome (AIDS). In the HIV/AIDS trial BEA reduced the incidence of TB co-infection by $42 \cdot 2 \%$ and the cumulative incidence of opportunistic infections (38).

In the present study a new water-miscible formulation of BEA was used. Its ability to form a stable suspension in water is derived from a new method of formulating the molecule. Water miscibility avoids the problems associated with the organic solvents required to administer the older, lipid soluble formulation. Mice treated with this new BEA formulation showed similar results as the old synthetic analog, producing significant increase of IFN- $\gamma$ and TNF- $\alpha$, in coexistence with pulmonary bacillary loads reduction, and now

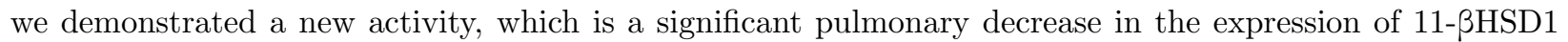

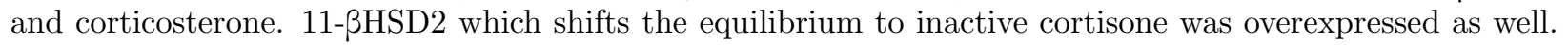
Thus, BEA can induce CD4 Th1 cells and macrophages activation by direct activity, and as shown in the present work, by the suppression of the local production of corticosterone in the lungs.

Obesity is commonly associated with T2D and other manifestations of the metabolic syndrome [1,2]. The metabolic syndrome manifests as obesity, hyperlipidemia and T2D, and is related to insulin resistance in adipose tissue, skeletal muscle and liver $[1,3]$. In obesity and T2D, GCs are important pathogenic factors because they are antagonists of insulin action. GC impair glucose uptake, enhance lipolysis and hepatic gluconeogenesis and promote proteolysis. Moreover, GCs directly inhibit pancreatic secretion of insulin, enhance glucose secretion by inhibiting gluconeogenesis in the liver and oppose other metabolic actions, such as insulin signaling and glucose uptake by inhibiting the translocation of the glucose transporter GLUT4 to the plasma membrane (19).

Our results show that in comparison with healthy control animals, mice with T2D without Mtb infection exhibited higher 11-3HSD1 expression and strong corticosterone immunostaining in bronchial epithelial cells and in hepatocytes. BEA treatment decreased the expression of both enzyme and hormone in lung and liver and corrected glucose concentration in blood. Thus, BEA significantly improved metabolic abnormalities by down-regulating 11-3HSD1 gene expression as one of the mechanisms. These results are in agreement with previous publications, and contribute new information such as the increase of corticosterone production in the airways epithelium of T2D individuals which could be a factor for higher predisposition of respiratory infections. One of these infections is TB.

Our results demonstrated a higher pulmonary bacillary load in T2D/TB than in TB mice without T2D, as

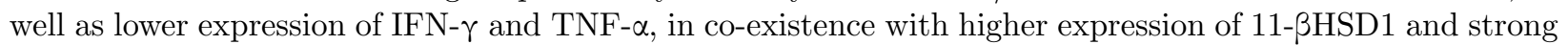
immunostaining to corticosterone in the pulmonary inflammatory intra-alveolar infiltrate, particularly in foamy macrophages. Thus, it seems that the higher pulmonary production of corticosterone mediated by $11-\beta H S D 1$ is a factor that worsens the course of TB in T2D and this could be a new therapeutic target.

In this regard, our results suggest that BEA can block 11-ßHSD1 in the lung and in the liver, becoming an effective treatment for this co-morbidity. Trials for the use of BEA in the treatment of human TB, T2D and the co-morbidity T2D/TB are now justified.

\section{Acknowledgments}

LT-MO is a PhD student from Programa de Doctorado en Ciencias Biomédicas, Universidad Nacional 
Autónoma de México (UNAM) and received fellowship 433346 from CONACyT. This work was sponsored by Consejo Nacional de Ciencia y Tecnología, Grant/Award Number: FC2015-1/115.

\section{Conclusion}

The study of T2D-TB comorbidity from the immunoendocrine point of view is of utmost importance to understand the background of this binomial, as well as to propose better strategies for the control of this comorbidity. Active GCs represent a negative factor in the development of both entities, with a negative effect on glucose metabolism and the development of a non-protective immune response. Treatment with BEA represents a novel strategy as an adjuvant in the treatment of TB alone and comorbidity with T2D. For these reasons, it is of utmost importance to continue with the study of the BEA through its escalation to clinical studies to be used in patients.

\section{Conflict of interest}

We declare not conflict of interest during the course of this work.

\section{Author contributions}

LT-MO, AC and HP-R contributed to the background work and conceived the experiments. LT-MO and RE-O performed, organized, and analyzed the results. LT-MO, B-E, EB and MC-B experiments design and execution. BP-J animal infections and drug administration. ME-D culture and preparation of Mycobacterium tuberculosis H37Rv strain to infection and CFU experiments, IT, NT, AT designed the hypercaloric diet. C-W, G-Y prepare and supply BEA for the animal treatment.

\section{References}

1. Ayelign B, Negash M, Genetu M, Wondmagegn T, Shibabaw T. Immunological Impacts of Diabetes on the Susceptibility of Mycobacterium tuberculosis. J Immunol Res (2019) 2019 : doi:10.1155/2019/6196532

2. Ehlers S, Schaible UE. The granuloma in tuberculosis: Dynamics of a host-pathogen collusion. Front Immunol (2012) 3 :1-9. doi:10.3389/fimmu.2012.00411

3. Meenakshi P, Ramya S, Lavanya J, Vijayalakshmi V, Sumanlatha G. Effect of IFN- $\gamma$, IL-12 and IL10 cytokine production and mRNA expression in tuberculosis patients with diabetes mellitus and their household contacts. Cytokine (2016) 81 :127-136. doi:10.1016/j.cyto.2016.03.009

4. Cooper AM, Mayer-Barber KD, Sher A. Role of innate cytokines in mycobacterial infection. Mucosal Immunol (2011) 4 :252-260. doi:10.1038/mi.2011.13

5. Boillat-Blanco N, Tumbo AMN, Perreau M, Amelio P, Ramaiya KL, Mganga M, Schindler C, Gagneux S, Reither K, Probst-Hensch N, et al. Hyperglycaemia is inversely correlated with live M. bovis BCG-specific CD4+ T cell responses in Tanzanian adults with latent or active tuberculosis. Immun Inflamm Dis (2018) 6 :345-353. doi:10.1002/iid3.222

6. Hodgson K, Morris J, Bridson T, Govan B, Rush C, Ketheesan N. Immunological mechanisms contributing to the double burden of diabetes and intracellular bacterial infections. Immunology (2015)144:171-185. doi:10.1111/imm.12394

7. Rey A del, Mahuad C V., Bozza V V., Bogue C, Farroni MA, Bay ML, Bottasso OA, Besedovsky HO. Endocrine and cytokine responses in humans with pulmonary tuberculosis. Brain Behav Immun (2007)21 :171-179. doi:10.1016/j.bbi.2006.06.005

8. Fernández R, Díaz A, D’Attilio L, Bongiovanni B, Santucci N, Bertola D, Besedovsky H, del Rey A, Bay ML, Bottasso O. An adverse immune-endocrine profile in patients with tuberculosis and type 2 diabetes. Tuberculosis (2016) 101 :95-101. doi:10.1016/j.tube.2016.09.001

9. Talabér G, Jondal M, Okret S. Extra-adrenal glucocorticoid synthesis: Immune regulation and aspects on local organ homeostasis. Mol Cell Endocrinol (2013) 380 :89-98. doi:10.1016/j.mce.2013.05.007 
10. Loerz C, Maser E. The cortisol-activating enzyme 11ß-hydroxysteroid dehydrogenase type 1 in skeletal muscle in the pathogenesis of the metabolic syndrome. J Steroid Biochem Mol Biol (2017)174:65-71. doi:10.1016/j.jsbmb.2017.07.030

11. Gomez-Sanchez EP, Ganjam V, Chen YJ, Liu Y, Clark SA, Gomez-Sanchez CE. The $11 \beta$ hydroxysteroid dehydrogenase 2 exists as an inactive dimer.Steroids (2001) 66 :845-848. doi:10.1016/S0039-128X(01)001192

12. Chapman KE, Coutinho AE, Gray M, Gilmour JS, Savill JS, Seckl JR. The role and regulation of 11ß-hydroxysteroid dehydrogenase type 1 in the inflammatory response. Mol Cell Endocrinol (2009)301 :123-131. doi:10.1016/j.mce.2008.09.031

13. Cidlowski DCJA. One Hormone, Two Actions : Anti- and Pro-Inflammatory Effects of Glucocorticoids. (2015)27709 :20-32. doi:10.1159/000362724

14. Pandolfi J, Baz P, Fernández P, Discianni Lupi A, Payaslián F, Billordo LA, Fainboim L, Arruvito L. Regulatory and effector T-cells are differentially modulated by Dexamethasone. Clin Immunol (2013)149 :400-410. doi:10.1016/j.clim.2013.09.008

15. Sapolsky SFS and RM. An Inflammatory Review of Glucocorticoid Actions in the CNS. Bone (2012) 23 :1-7. doi:10.1016/j.bbi.2006.06.005

16. Masuzaki H, Paterson J, Shinyama H, Morton NM, Mullins JJ, Seckl JR, Flier JS. A transgenic model of visceral obesity and the metabolic syndrome. Science (80- ) (2001) 294 :2166-2170. doi:10.1126/science.1066285

17. Apostolova G, Schweizer RAS, Balazs Z, Kostadinova RM, Odermatt A. Dehydroepiandrosterone inhibits the amplification of glucocorticoid action in adipose tissue. Am J Physiol - Endocrinol Metab (2005)288 : doi:10.1152/ajpendo.00442.2004

18. Sävendahl L. The effect of acute and chronic stress on growth.Sci Signal (2012) $\mathbf{5}$ :9-11. doi:10.1126/scisignal.2003484

19. Shukla R, Basu AK, Mandal B, Mukhopadhyay P, Maity A, Chakraborty S, Devrabhai PK. 11ß Hydroxysteroid dehydrogenase - 1 activity in type 2 diabetes mellitus: A comparative study. BMC Endocr Disord (2019) 19 :1-9. doi:10.1186/s12902-019-0344-9

20. Chiodini I, Adda G, Scillitani A, Coletti F, Morelli V, Di Lembo S, Epaminonda P, Masserini B, BeckPeccoz P, Orsi E, et al. Cortisol secretion in patients with type 2 diabetes: Relationship with chronic complications. Diabetes Care (2007) 30 :83-88. doi:10.2337/dc06-1267

21. Buford TW, Willoughby DS. Impact of DHEA(S) and cortisol on immune function in aging: A brief review. Appl Physiol Nutr Metab (2008)33 :429-433. doi:10.1139/H08-013

22. Nicoletti F, Conrad D, Wang A, Pieters R, Mangano K, Van Heeckeren A, White SK, Frincke J, Reading CL, Auci DL, et al. 16 $\alpha$-Bromoepiandrosterone (HE2000) limits non-productive inflammation and stimulates immunity in lungs. Clin Exp Immunol (2009)158 :308-316. doi:10.1111/j.1365-2249.2009.04032.x

23. Ramos-Espinosa O, Islas-Weinstein L, Peralta-Álvarez MP, López-Torres MO, Hernández-Pando R. The use of immunotherapy for the treatment of tuberculosis. Expert Rev Respir Med (2018)12:427-440. doi:10.1080/17476348.2018.1457439

24. Hernández-Pando R, Orozcoe H, Sampieri A, Pavón L, Velasquillo C, Larriva-Sahd J, Alcocer JM, Madrid M V. Correlation between the kinetics of Th1, Th2 cells and pathology in a murine model of experimental pulmonary tuberculosis. Immunology (1996) 89 :26-33.

25. Segura-Cerda CA, Marquina-Castillo B, Lozano-Ordaz V, Mata-Espinosa D, Barrios-Payán JA, LópezTorres MO, Aceves-Sánchez M de J, Bielefeldt-Ohmann H, Hernández-Pando R, Flores-Valdez MA. BCG and 
BCG $\triangle$ BCG1419c protect type 2 diabetic mice against tuberculosis via different participation of $\mathrm{T}$ and $\mathrm{B}$ lymphocytes, dendritic cells and pro-inflammatory cytokines. npj Vaccines (2020) 5 :1-10. doi:10.1038/s41541020-0169-6

26. Luo J, Quan J, Tsai J, Hobensack CK, Sullivan C, Hector R, Reaven GM. Nongenetic mouse models of non-insulin-dependent diabetes mellitus.Metabolism (1998) 47 :663-668. doi:DOI: 10.1016/S00260495(98)90027-0

27. Rangel-Santiago JF, Baay-Guzman GJ, Duran-Padilla MA, Lopez-Bochm KA, Garcia-Romero BL, Hernandez-Cueto DD, Pantoja-Escobar G, Vega MI, Hernandez-Pando R, Huerta-Yepez S. A novel role of Yin-Yang-1 in pulmonary tuberculosis through the regulation of the chemokine CCL4.Tuberculosis (2016) 96 :87-95. doi:10.1016/j.tube.2015.10.013

28. Baay-Guzman GJ, Duran-Padilla MA, Rangel-Santiago J, Tirado-Rodriguez B, Antonio-Andres G, Barrios-Payan J, Mata-Espinosa D, Klunder-Klunder M, Vega MI, Hernandez-Pando R, et al. Dual role of hypoxia-inducible factor $1 \alpha$ in experimental pulmonary tuberculosis: Its implication as a new therapeutic target. Future Microbiol (2018)13:785-798. doi:10.2217/fmb-2017-0168

29. Zapata-Tarres M, Juarez-Villegas LE, Maldonado-Valenzuela A, Baay-Guzman GJ, Lopez-Perez T V., Cabrera-Muñoz L, Sadowinski-Pine S, Huerta-Yepez S. Expression of YY1 in Wilms tumors with favorable histology is a risk factor for adverse outcomes. Futur Oncol(2019) 15 :1231-1241. doi:10.2217/fon-2018-0764

30. Schmittgen TD, Livak KJ. Analyzing real-time PCR data by the comparative CT method. Nat Protoc (2008) 3 :1101-1108. doi:10.1038/nprot.2008.73

31. Hernandez-Pando R, Orozco H, Aguilar D. Factors that deregulate the protective immune response in tuberculosis. Arch Immunol Ther Exp (Warsz) (2009) 57 :355-367. doi:10.1007/s00005-009-0042-9

32. Bongiovanni B, Díaz A, D’Attilio L, Santucci N, Dídoli G, Lioi S, Nannini LJ, Gardeñez W, Bogue C, Besedovsky $\mathrm{H}$, et al. Changes in the immune and endocrine responses of patients with pulmonary tuberculosis undergoing specific treatment. Ann N Y Acad Sci (2012)1262 :10-15. doi:10.1111/j.1749-6632.2012.06643.x

33. Hernandez-Pando R, Orozco H, Honour J, Silva P, Leyva R, Rook GAW. Adrenal changes in murine pulmonary tuberculosis; a clue to pathogenesis? FEMS Immunol Med Microbiol (1995)12:63-72. doi:10.1016/0928-8244(95)00051-8

34. Barrios-Payán J, Revuelta A, Mata-Espinosa D, Marquina-Castillo B, Villanueva EB, Gutiérrez MEH, Pérez-Sánchez G, Pavón L, Hernandez-Pando R. The contribution of the sympathetic nervous system to the immunopathology of experimental pulmonary tuberculosis. J Neuroimmunol (2016) 298 :98-105. doi:10.1016/j.jneuroim.2016.07.012

35. Irwin MR. Human psychoneuroimmunology: 20 Years of discovery.Brain Behav Immun (2008) 22 :129139. doi:10.1016/j.bbi.2007.07.013

36. Hernandez-Pando GAWR and R. Pathogenic Role, in Human and Murine Tuberculosis, of Changes in the Pheripheral Metabolism of Glucocorticoids ans Antiglucocorticoids. (1997) $22: 1-5$.

37. Streber, M de la Luz, Orozco H, Arriaga K, Pavon L. The effects of androstenediol and dehydroepiandrosterone on the course and cytokine profile of tuberculosis in BALB/c mice. Immunology(1998)234-241.

38. Leenstra T, ter Kuile FO, Kariuki SK, Nixon CP, Oloo AJ, Kager PA, Kurtis JD. Dehydroepiandrosterone Sulfate Levels Associated with Decreased Malaria Parasite Density and Increased Hemoglobin Concentration in Pubertal Girls from Western Kenya. J Infect Dis(2003) 188 :297-304. doi:10.1086/376508

39. Bozza V V., D’Attilio L, Mahuad C V., Giri AA, Del Rey A, Besedovsky H, Bottasso O, Bay ML. Altered cortisol/DHEA ratio in tuberculosis patients and its relationship with abnormalities in the mycobacterialdriven cytokine production by peripheral blood mononuclear cells. Scand J Immunol (2007) 66 :97-103. doi:10.1111/j.1365-3083.2007.01952.x 


\section{Figures}
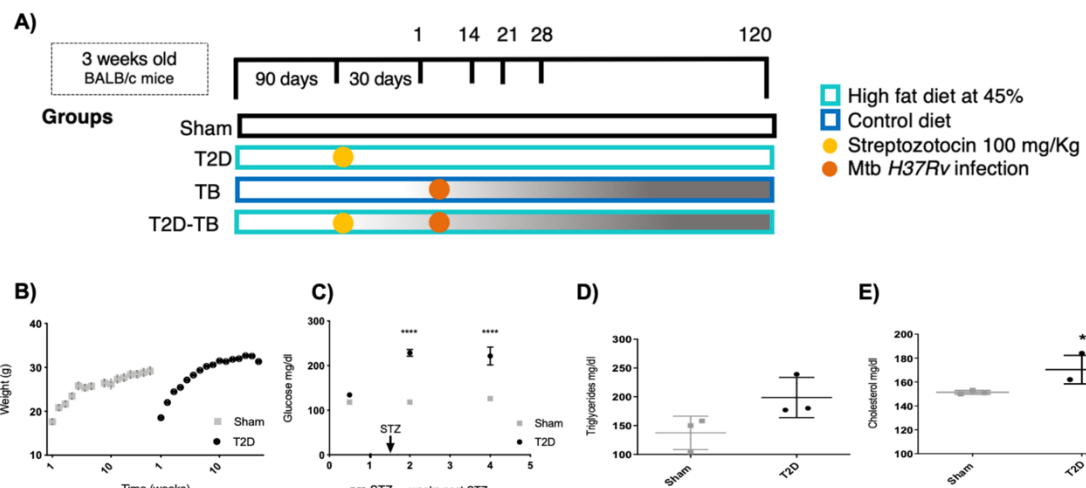

D)
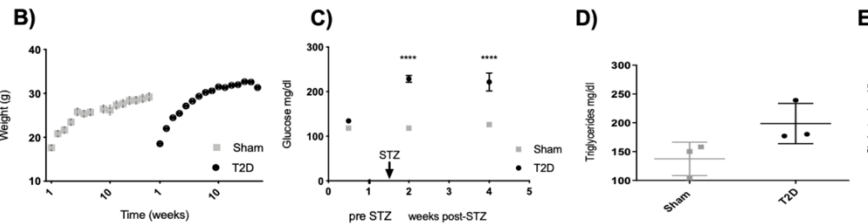

E)

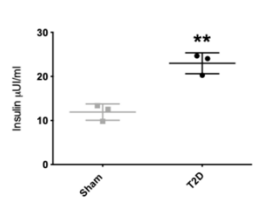

)

G)

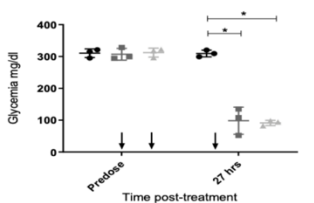

H)

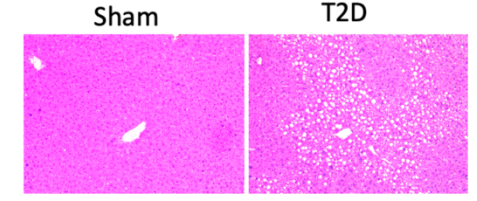

Figure 1.- Metabolic abnormalities and liver pathology of mice with type 2 diabetes mellitus model vs non-diabetic animals (control).A) General description of the experimental groups. B) mice were weighted weekly to monitor the weight gain induced by the hypercaloric diet. Data are presented as a mean and standard derivation of 5 mice per group for 16 weeks. C) Blood glucose levels were assessed two and four weeks after administration of STZ intraperitoneally in animals fed with a high-caloric diet treated with streptozotocin (HFD-STZ) and in animals not treated with STZ and fed with Chow control diet. Concentrations in sera of C) triglyceride, D) cholesterol, and E) insulin in control and T2D groups, after four months of feeding with hyper caloric diet and one month of STZ treatment. G) Effect of hypoglycemic drugs on the control of serum glucose levels in T2D animals, before and after 8 and 24 hours of oral administration of metformin (Met) or glibenclamide (Gly), arrows indicate administration times $(0,8$ and 24 hours). Asterisk represents statistical significance (one-way ANOVA, $\mathrm{P}<0.05$, and two-way ANOVA for graph $1 \mathrm{~B}, 1 \mathrm{C}$, and $1 \mathrm{G}$ ).H) Representative micrograph of liver tissue from a control mouse (left figure) and extensive cytoplasmic vacuolization of hepatocytes corresponding to steatosis in T2D mouse (right figure). 
A)

C)
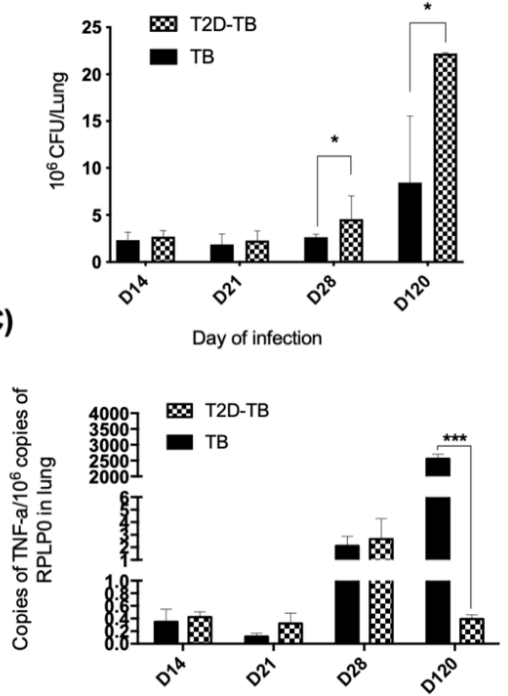

B)

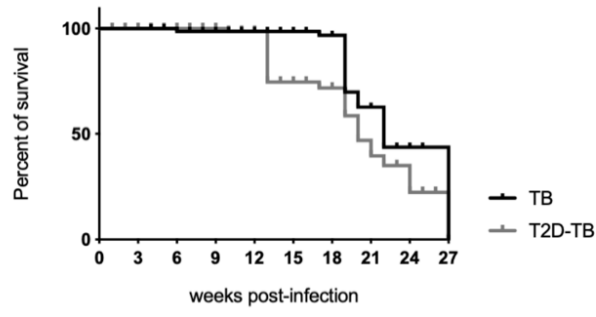

Figure. 2. Comparative course of pulmonary tuberculosis in diabetic (T2D-TB) and nondiabetic (TB) mice. A) Five mice per group were sacrificed at the indicated time points $(14,21,28$, and 120 post-infection) and the right lungs were used to determine the bacterial loads by counting colony-forming units (CFU). A significant increase in the bacillary load of the T2D-TB group was observed after one and four months of infection in comparison with the TB group. B) The comparative survival rate of both groups. C) Comparative expression of TNF $\alpha$ determined by RT-PCR in the lungs of T2D/TB and TB groups, there is significant lower expression after 120 days of infection in the former group. Asterisk represent statistical significance $\mathrm{p}<0.05$.

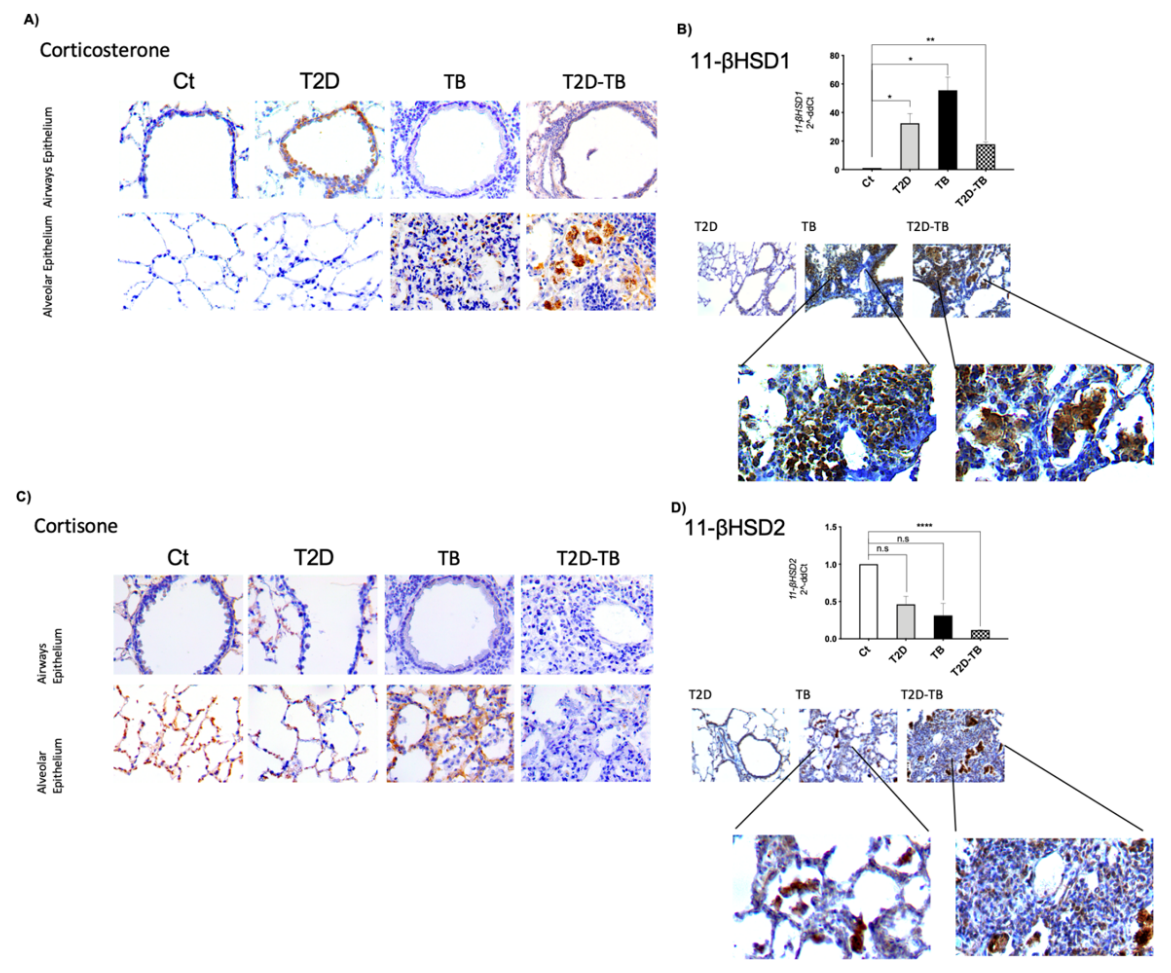


Figure 3.- Gene expression and cellular sources of glucocorticoids and their converting enzymes in late TB (day 120). A) Representative micrographs of immunohistochemical detection of corticosterone in the epithelium of the lung airways and the alveolar epithelium. Occasional bronchial epithelial cells show slight positivity in the control mouse $(\mathrm{CT})$, while in T2D mouse numerous bronchial cells are strongly positive. Bronchial epithelium is negative in TB, and T2D-TB; while in the pneumonic areas numerous macrophages show intense positivity. B) Top panel show the comparative gene expression of 11- $\beta$ HSD1 determined by qPCR in the different experimental groups at day 120 . T2D, TB, and T2D-TB groups show a significant increase in comparison with the control group. Bottom panel show immunohistochemical detection of 11- $\beta$ HSD1, foamy vacuolated macrophages (inset) in pneumonic areas from TB and T2D-TB show strong immunostaining. C) Representative micrographs of immunohistochemistry detection of cortisone in bronchial epithelial cells and alveoli. Alveolar epithelium show positive immunostaining in the control mouse, while bronchial cells are negative in all the conditions. TB mouse show strong immunostaining in alveolar epithelium and in occasional inflammatory cells, while T2D and T2D/TB are negative. D) Comparative gene expression of 11-3HSD2 and its representative immunohistochemistry detection. Asterisks represent statistical significance (**** $\mathrm{p}<0.0001$, two- way ANOVA and n.s. no significance). 


\section{Lung}

TB

T2D-TB

A)
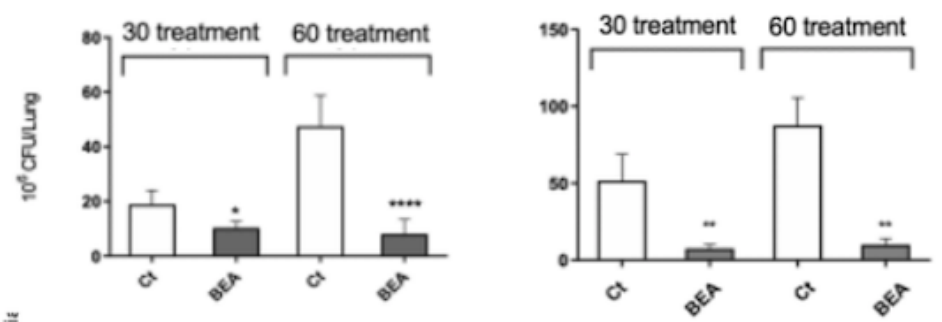

B)
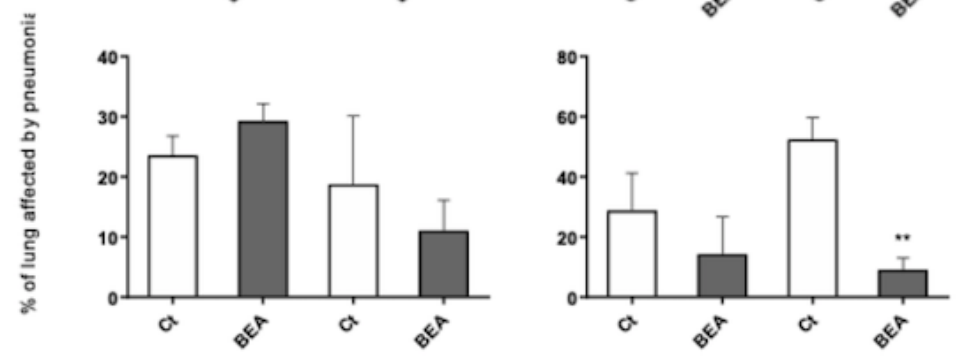

C)
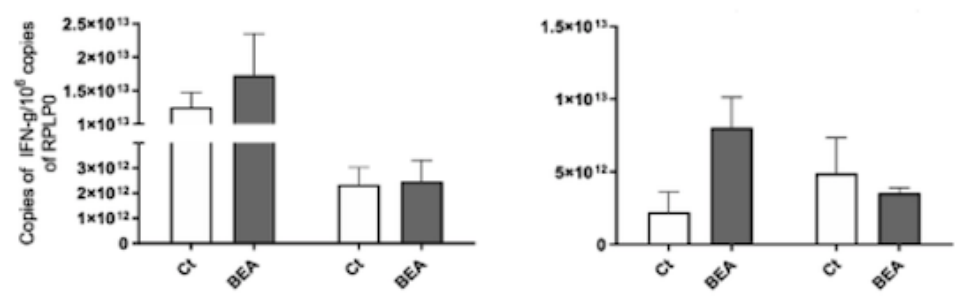

D)
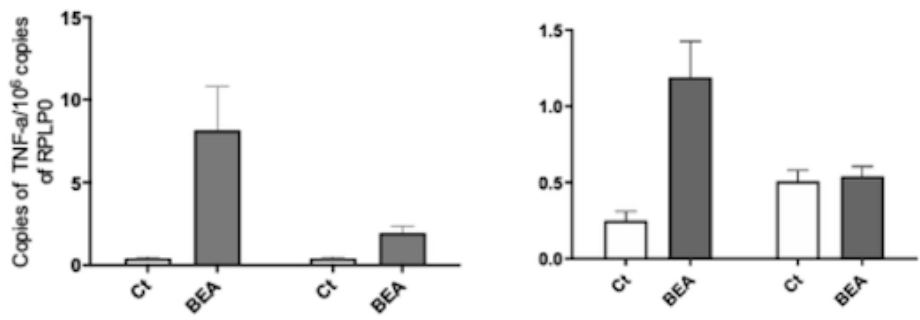

\section{Spleen}

E)
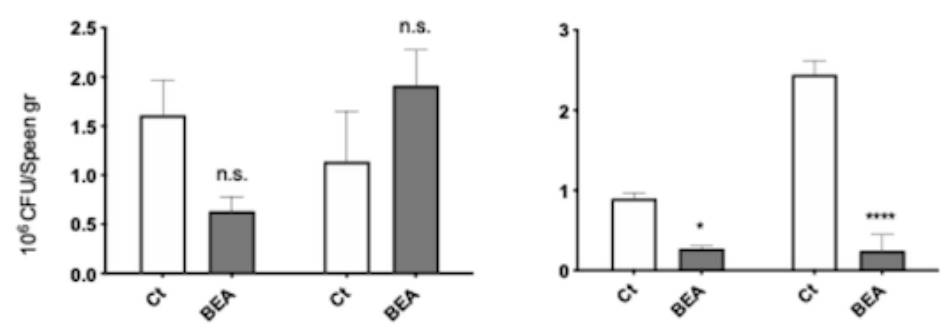

F)
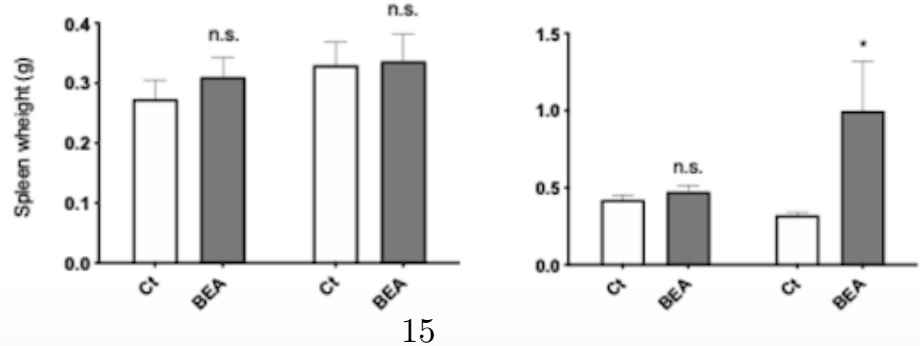
Figure 4.- Therapeutic effect of BEA in TB and T2D / TB mice.Groups of TB and T2D/TB mice after 60 days of intratracheal infection with high dose Mtb strain H37Rv were treated during one or two months with BEA, while the control group received only the vehicle. Lungs were processed for the indicated determination. In comparison with the control mice, in both TB and T2D/TB groups and particularly after 60 days of treatment, BEA induced significant decrease of pulmonary bacillary loads and tissue damage (pneumonia), while after one month of treatment BEA induced higher expression of the protective cytokines $\mathrm{TNF} \alpha$ and IFN- $\gamma$. In the spleen, treatment with BEA did not produce significant changes in the weight and CFUs in TB mice, while in T2D/TB mice induced after two months of treatment significant decrease of bacillary burdens and higher weight increase. Asterisks represent statistical significance $(* * * * \mathrm{p}<0.0001$, two- way ANOVA and n.s. no significance).
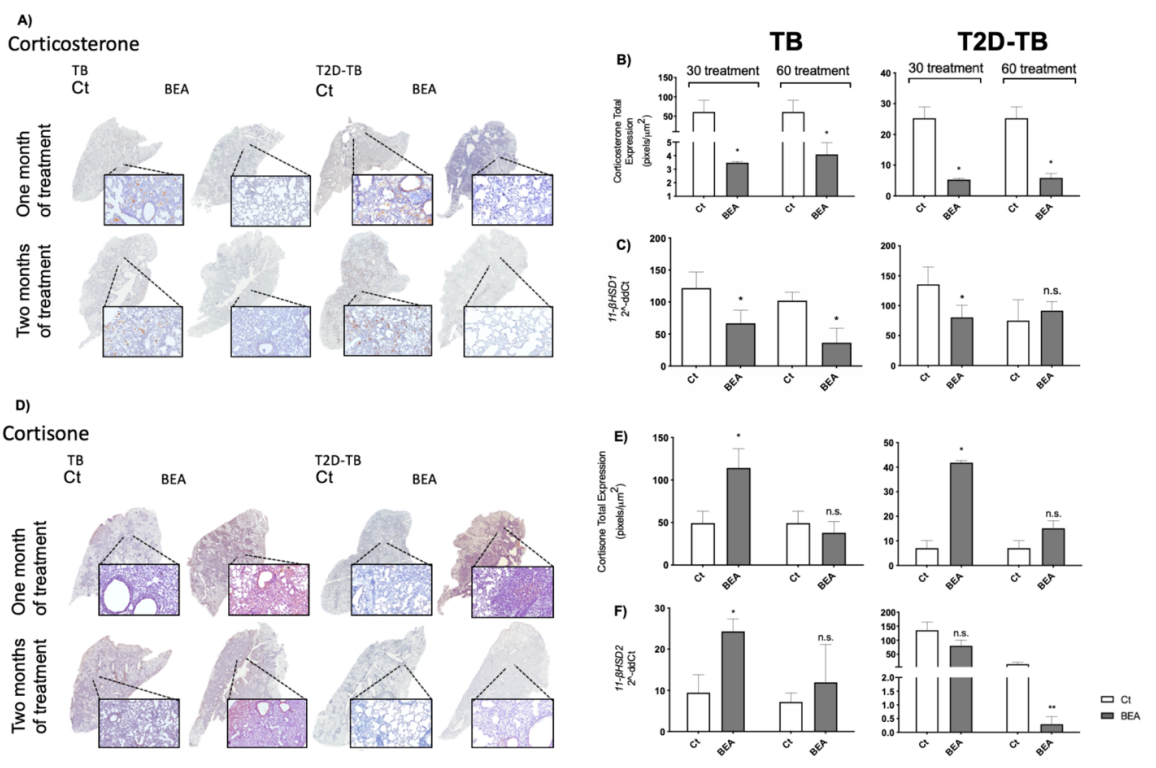

Figure 5.- Effect of BEA on lung glucocorticoids production and the gene expression of their converting enzymes in TB and T2D-TB mice.A) Representative low power and mild power micrographs of immunohistochemistry detection of corticosterone, in both groups after one and two months of BEA treatment. B) In comparison with the control group, the digital pathology analysis showed significant lower immunostaining of corticosterone induced by BEA treatment after one and two months in TB mice and T2D-TB mice. C) BEA treatment induced lower 11b-HSD1 gene expression in TB and T2D-TB in comparison with vehicle-treated animals. D) Representative micrographs of cortisone detection by immunohistochemistry in the lungs of TB and T2D mice treated with BEA at one and two months of infection. E) The digital pathology study shows higher expression of cortisone after one month of treatment in the TB and T2D/TB groups. F) Gene expression of 11b-HSD2 corticosterone converting enzyme after BEA treatment in TB and T2D-TB animals. Asterisk represents statistical significance $(\mathrm{p}<0.05)$. 
T2D

A)

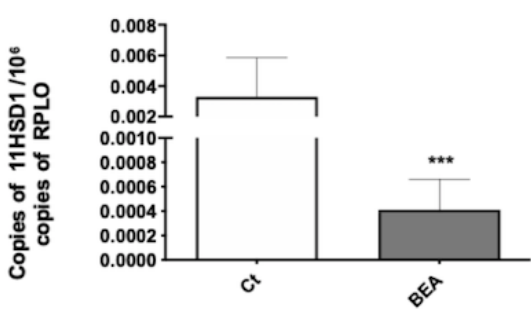

B)

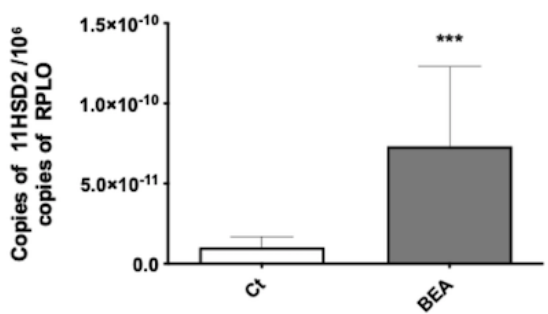

C)

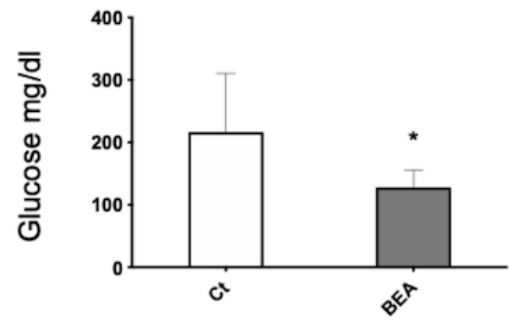

D)

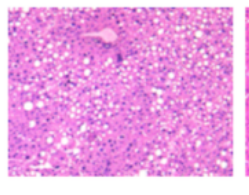

c

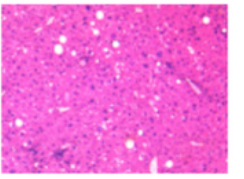

$6^{8}$
T2D-TB
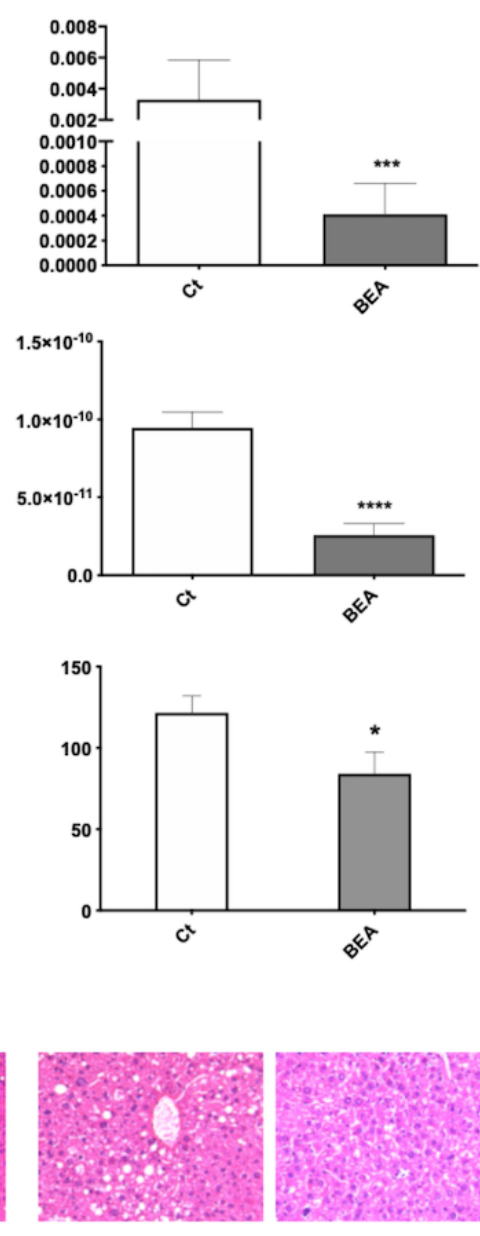

$c^{2}$

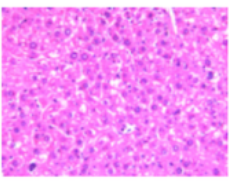

$8^{8}$

Figure 6.- Effect of BEA in the liver of T2D and T2D-TB mice.Liver samples from groups of five T2D and T2D-TB mice treated with BEA for one month and their respective control mice that received

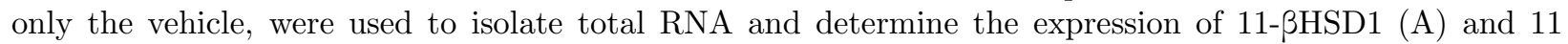
- $\beta$ HSD2 (B) by RT-PCR. C) Treatment with BEA corrected hyperglycemia in T2D and T2D-TB mice. Asterisks represent statistical significance ${ }^{* * *} \mathrm{p}<0.0001$, ${ }^{* * * *} \mathrm{p}<0.0001$, two-way ANOVA, n.s. without significance). D) Liver tissue sections stained with $\mathrm{H} / \mathrm{E}$ show steatosis in the control mouse, which was eliminated by treatment with BEA in T2D and T2D-TB mice. 